Pesq. Vet. Bras. 37(6):603-607, junho 2017 DOI: $10.1590 / \mathrm{S} 0100-736 \mathrm{X} 2017000600012$

\title{
Fipronil/(S)-methoprene spot-on to control fleas on cats in a field trial in Spain ${ }^{1}$
}

\author{
María J. Gracia ${ }^{2 *}$, Carlos Calvete ${ }^{3}$, Rosa Estrada², José M. Marcén², Rocío Pinal ${ }^{2}$ \\ and Miguel A. Peribáñez
}

\begin{abstract}
Gracia M.J., Calvete C., Estrada R., Marcén J.M., Pinal R. \& Peribáñez M.A. 2017. Fipronil/(S)-methoprene spot-on to control fleas on cats in a field trial in Spain. Pesquisa Veterinária Brasileira 37(6):603-607. Departamento de Patología Animal, Parasitología, Facultad de Veterinaria, Calle Miguel Servet 177, 50013 Zaragoza, España. E-mail: mjgracia@unizar.es

The study was conducted in order to evaluate the effect of a fipronil/(S)-methoprene formulation against fleas on naturally infested cats. The study involved a population of 89 cats distributed among 24 veterinary practices in 9 regions of Spain. The product was applied according to label instructions on days 0,30 and 60. Animals underwent parasitological and clinical assessments on day 0 and thereafter in monthly intervals (every 30 days) until day 90. Ctenocephalides felis was the most abundant species $(98.9 \%$ of all fleas collected), and flea abundance on Day 0 was associated with the hair type, the location of the household, and the time elapsed from the last anti-flea treatment. Fipronil/(S)-methoprene demonstrated high efficacy and induced the reduction of clinical signs related to the presence of fleas. Clinical signs and flea abundance decreased significantly throughout time (P=0.001) with an efficacy rate of $72.6 \%$ at Day $30,88.4 \%$ at Day 60 and $93.9 \%$ at Day 90. A high level of flea control and a remission of the clinical signs related to presence of fleas were observed on cats following 3 monthly applications a fipronil/(S)-methoprene formulation.
\end{abstract}

INDEX TERMS: Feline, ectoparasites, Ctenocephalides felis, fipronil, (S)-methoprene.

\section{INTRODUCTION}

Fleas are the most common ectoparasites in cats and are present in many areas of the world, with Ctenocephalides felis Bouché, 1835, being the most frequent flea species on cats followed by Ctenocephalides canis (Cadiergues et al. 2000, Beck et al. 2006, Bond et al. 2007, Gracia et al. 2013).

Cat fleas play an important role as vectors. C. felis is the major vector of Bartonella spp., including Bartonella henselae, the agent of cat-scratch disease (CSD) (Chomel et al. 2006) and acts both as a reservoir host and as a vector for Rickettsia felis, the major agent of flea-borne spotted fever which is an emerging clinical disease of humans worldwide (Pérez-Osorio et al. 2008). The natural route of transmission of feline haemoplasma infection (Mycoplasma hae-

\footnotetext{
${ }^{1}$ Received on October 21, 2015.

Accepted for publication on October 16, 2016.

${ }^{2}$ Departamento de Patología Animal, Facultad de Veterinaria, Parasitología, Universidad de Zaragoza, Calle Miguel Servet 177, 50013 Zaragoza, España. *Corresponding author: mjgracia@unizar.es

${ }^{3}$ Unidad de Sanidad Animal. Centro de Investigación y Tecnología Agroalimentaria (CITA), Av. Montañana 930, 50059-Zaragoza, España.
}

mofelis) has not been confirmed, but fleas are implicated (Tasker 2010). Additionally, cat fleas are the intermediate host of Dipylidium caninum. Simple pruritus or Flea allergic dermatitis (FAD) is a very important consequence of flea infestation. Signs of this reaction include pruritus, erythema, papules, pustules and crusts (scabs). Also if severe, hair loss and anemia may occur. Therefore, effective control measures against these parasites are important in preventing feline and human diseases.

Fipronil and (S)-methoprene have different modes of action against insects. Fipronil is a broad spectrum phenylpyrazole with activity against both adult insects and arachnids; moreover, it exhibits a high selectivity to insects over mammals (Narahashi et al. 2007, Narahashi et al. 2010). (S)-methoprene is an insect growth regulator (IGR) causing impairment of development and death of insect larval stages. (S)-methoprene is present in the skin lipids and penetrates into flea eggs before they fall off the animals, providing high and persistent activity against immature stages of the cat flea by interfering with the "in situ" development of eggs and subsequent inhibition of adult flea emergence (Young et al. 2004). Fipronil and (S)-methoprene have been 
used in combination for the treatment and control of fleas, for several years (Dryden et al. 2007, Schnieder et al. 2008, Beugnet \& Franc 2010).

One potential reason for failure of a flea control program is resistance of the insects to the chemicals used. Although resistance to old insecticides including cyclodienes, carbamates, organophosphates, and pyrethroids has been demonstrated in fleas, (Bossard et al. 2002) resistance against modern adulticides in field strains has not. Because resistance selection is certainly possible in fleas, it is crucial that we attempt to know the efficacy of the current therapeutic agents.

The present work was conducted in order to evaluate the efficacy of a fipronil/(S)-methoprene formulation (Frontline Combo $^{\circledR}$ Spot-on, Merial, France) against fleas on cats naturally infested. In addition, observations also were made on the beneficial effect of treatment on cats showing clinical signs of flea infestation, including animals with signs of flea allergy dermatitis (FAD).

\section{MATERIALS AND METHODS}

Area of study and sample collection. The survey was carried out on a population of 89 cats distributed amongst 24 veterinary practices in 9 regions of Spain (Fig.1). The veterinary practices collaborated voluntarily in the survey and only cats parasitized by at least one flea at day 0 and untreated for, at least two months prior to entry into the study were used. The number of cats ranged from 1 to 8 per practice, enrolled by means of a random selection among cat owners. The study took place from April 2010 to August 2011.

After the informed owner consent had been obtained, animals were recruited in the study. Data recorded from each cat include age, sex, breed and hair type.

Parasitological and clinical evaluations. Day 0 was defined individually as the day the animal was found suitable for recruitment in the study. On day 0 , the appropriate parasitological and clinical examinations were carried out.

The examining veterinarian performed a flea count according to a defined procedure (Marchiondo et al. 2007). The cat was combed with a fine-toothed comb over the entire body for 7 minutes including dorsal midline ( $2 \mathrm{~min}$ ), tail head ( $2 \mathrm{~min}$ ), left lateral (1 min), right lateral (1 min) and inguinal (1 min). In case of collecting at least one flea, the combing was continued for an extra minute over each section. After obtaining the agreement from the owner, fleas were put back on the cat in order to mimic as much as possible the natural parasitation status of the animal, and the real situation of a treated infestation. The same protocol was performed on days 30,60 and 90. A small sample of fleas obtained on day 0 , were stored at $-20^{\circ} \mathrm{C}$ and transferred to the Department of Animal Pathology (Zaragoza University, Spain) for identification purposes (Beaucournu \& Launay 1990).

A clinical examination was performed on each cat and dermatological lesions and abrasions related to the presence of fleas (typical FAD sores, scratching and flea bites) as well as flea faeces were also recorded. Flea allergy dermatitis was diagnosed on the basis of clinical signs and the animal's history.

Treatment. When the parasitological and clinical evaluations were completed, cats were treated. Each cat was treated with Frontline Combo ${ }^{\circledR}$ by the veterinarian, following the product labeling. Treatments were performed on days 0, 30 and 60 .

Additional information. A questionnaire was used to collect other data regarding the cats:

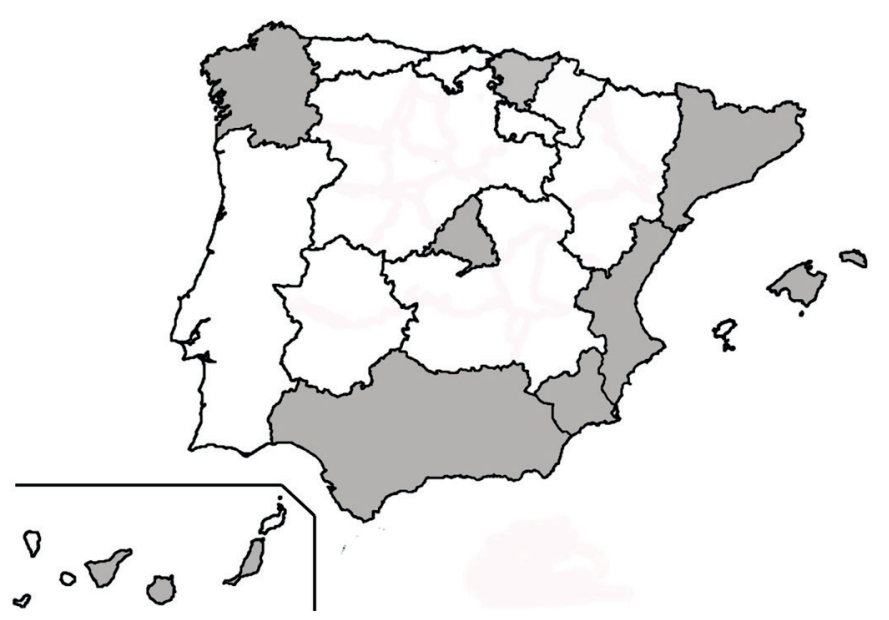

Fig.1. Map of Spain showing the location of regions where the cats were sampled.

Location of the household: considered urban when located in a town or in the center of a village, or surrounded entirely by urbanized area; considered rural/suburban when located in the countryside or in partially urbanized areas.

Outside activity: cats were classified according to their activity as either partially or totally outdoors (access to a garden, street or other outside environments) or entirely indoors.

Multi-pet households: whether residence was shared with other pets.

Contact with other pets: whether cats were in contact with other pets in the neighborhood.

Period of treatment during the year: whether the cat was treated for the first time in the warm period (May-October) or in the cold period (November-April).

Information related to previous treatments was also recorded: product and frequency of use.

The cats underwent parasitological and clinical assessments on days $0,30,60$ and 90 . Even though 89 cats started the study, only 75 cats could be followed for the whole period of study.

Statistical analysis. As the sample size decreased from day 0 to day 90 , two separate analyses were performed. In first, data obtained on Day 0 were used to explore the relationships between flea infestation and host, habitat and management factors recorded at the beginning of the survey (Day 0). In order to achieve this, a General Linear Model was set up, in which the log-transformation $(\operatorname{Ln}(x+1))$ of flea abundance in each cat on Day 0 was used as dependent variable whereas next variables collected in the questionnaire were used as predictor variables: age (in months), sex, hair type, location of the household, outside activity, multipet households, contact with other pets, period of treatment in the year and time elapsed from last treatment (in months). Categorical predictor variables were coded as dummy variables. No interactions among predictors were included in the initial model. A backward selection procedure was used to obtain the final fitted model.

Secondly, data obtained from cats that remained enlisted in the survey until Day 90 were used to test the efficacy of treatment over time (from day 0 to day 90 ) and the possible effects of host, habitat and management factors. In this case, a General Linear Model (Mardia et al. 1979) with repeated measures was fitted to data. Flea abundance on days $0,30,60$ and 90 was used as a log-transformed $(\operatorname{Ln}(\mathrm{x}+1))$ dependent variable, whereas the same predictor variables as in the former analysis were included in the initial model. To explore the effects of predictor variables on treatment efficacy, second grade interactions among them and 
time were included in the initial model. A backward selection procedure was used to refine the final one. Post-hoc comparisons of log-transformed flea abundance over time were performed by Bonferroni test (Cabin \& Mitchell 2000).

Efficacy, as percentage reduction in mean flea infestation burden, was assessed over time by comparing mean infestation burdens on day 30,60 and 90 with mean flea burden registered at day 0 . The percentage reduction was $100(1-\mathrm{Xt} / \mathrm{Xc})$ where $\mathrm{Xc}$ was the arithmetic mean flea burden at day 0 and Xt was the arithmetic mean infestation burden on days 30,60 or 90. Following the World Association for the Advancement of Veterinary Parasitology (W.A.A.V.P.), the arithmetic mean was preferable to the geometric mean as it is easier to calculate, it provides a better estimate of count outputs and it is a more conservative measure of efficacy (Coles et al. 1992).

One tail Chi-square test was used to perform pair comparisons of the number of cats with fleas, dermatological lesions, lesions related to the presence of fleas (typical FAD lesions, scratching and flea bites) and flea faeces among the four sampling periods, i.e. days $0,30,60$ and 90 .

\section{RESULTS}

Among the 89 cats there were 47 males and 42 females. The age distribution is shown in Figure 2. Most cats were European (57 cats), followed by mixed breeds (15), Siamese (9) and Persian (8). 71 cats had short hair, 10 medium and 8 long hairs. Sixty-three cats (70.8\%) were surveyed in the warm period (May-October) and twenty six (29.2\%) in the cold period (November-April). Information about aspects related to previous treatments (product and frequency of use) showed that the owners of 43 cats out of 89 cats studied, ( $48.3 \%)$ remembered the trademark name of the product used to control fleas, while for 46 cats (51.7\%) the owner was unaware of this information. The active ingredients used were mainly fipronil $(83.7 \%$, of which $11 \%$ combined with (S)-methoprene, followed by selamectin (11.6\%), and imidacloprid (2.3\%). Among 41 owners who gave information about frequency of treatments against fleas, $46.3 \%$ treated every 1-3 months throughout the whole year, $7.3 \%$ treated every 3-6 months throughout the whole year, 19.5 $\%$ treated only during summer time and $26.8 \%$ treated when the owner considered it appropriate.

A total of 463 fleas were collected, representing two species: C. felis and Echidnophaga gallinacea, C. felis was the most abundant (458 fleas, $98.9 \%$ of the total), while $E$. gallinacea was only recorded from one cat (5 fleas, $1.1 \%$ of the total) located in Fuerteventura, Canary Islands.

On day 0 , a total of 89 cats were enrolled. The minimum infestation (1-4 fleas) was found in 20 cats, while most cats (52 animals) were infested with 5 to 10 fleas, and 17 animals were infested with more than 10 fleas (maximum of 50 fleas in a cat).

Flea abundance at Day 0 (Table 1) was associated with hair type, location of the household and time elapsed from the last treatment $\left(R^{2}=0.45 ; F=13.64 ; P<0.001\right)$. Flea burden was higher in cats with long hair $(P<0.001)$ compared with cats with medium or short hair. Flea burden was lower in cats living in urban areas $(P=0.022)$ in comparison to cats living in rural areas. In addition, a positive relationship was found between flea abundance and time elapsed from the last treatment used against fleas $(P=0.007)$. No statis- tically significant $(P>0.05)$ association was found with the other factors studied.

\section{Efficacy of treatment}

From the 75 animals that were surveyed during the 90 days, the percentage of flea free cats increased from $0 \%$ on day 0 to $76 \%$ on day 90 (Chi square $=237.5 ; \mathrm{P}<0.001$ ). The percentage reduction of mean flea burden was $72.6 \%$ on day $30,88.4 \%$ on day 60 and $93.9 \%$ on day 90 when compared to the mean flea burden registered at day 0 (Table 2 ). The statistical analysis confirmed that flea abundance decreased significantly over time $(F=114.38 ; P<0.001)$ and that decrease was statistically significant (Bonferroni test: $P<0.01$ ) among all sampling periods, excepting between days 60 and 90 , when no statistical difference was detected $(P=0.368)$.

The monthly treatments were effective to reduce clinical signs related to presence of fleas; dermatological lesions (including typical FAD lesions, scratching, and flea bites) and presence of flea faeces decreased throughout the study. On day 0 , before treatment was applied, of 75 cats that remained enlisted in the survey until day 90, 20 ani-

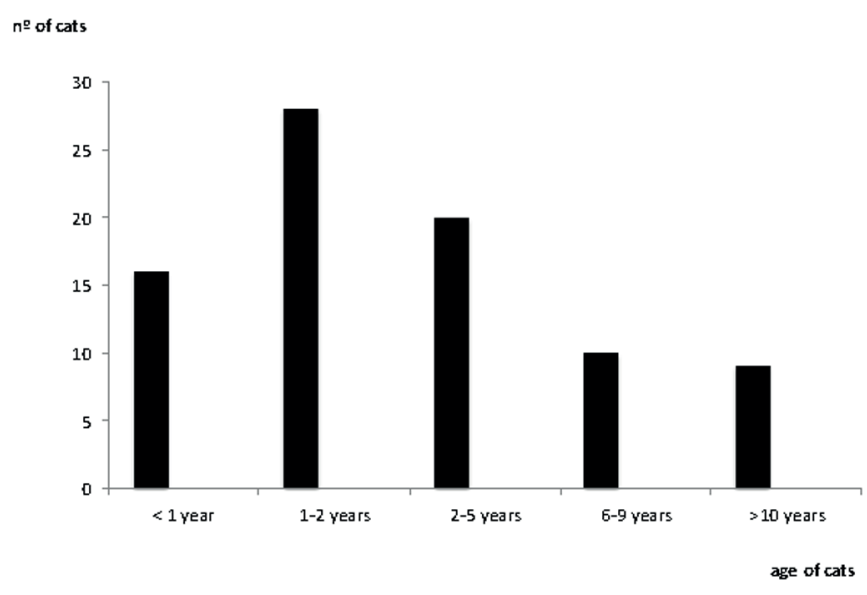

Fig.2. Age distribution of the treated cats.

Table 1. Number of cats $(\mathrm{N})$, arithmetic mean of fleas \pm SD and ranges based on hair type, location of household and time elapsed since last treatment

\begin{tabular}{lccccc}
\hline & & $\mathrm{N}$ & Arithmetic Mean & SD & range \\
\hline \multirow{2}{*}{ Hair type } & Short hair & 80 & 7.79 & 8.07 & $1-50$ \\
& Long hair & 8 & 20.87 & 15.72 & $5-50$ \\
Household & Rural/suburban & 47 & 9.47 & 9.18 & $1-50$ \\
& Urban & 42 & 8.31 & 10.17 & $1-50$ \\
Elapsed time since & 2-4 months & 24 & 5.42 & 3.52 & $1-13$ \\
the last treatment & $5-8$ months & 12 & 8.08 & 5.76 & $3-20$ \\
& $\geq 9$ months & 39 & 10.15 & 9.63 & $1-50$
\end{tabular}

Table 2. Prevalence of infestation, arithmetic mean \pm SD, minmax of fleas, efficacy (\%) and $95 \%$ CI from day 0 to day 90

\begin{tabular}{lccccc}
\hline & $\begin{array}{c}\text { Prevalence } \\
\text { of infestation }\end{array}$ & Mean \pm SD & Min-max & $\begin{array}{c}\text { Efficacy } \\
(\%)\end{array}$ & $\begin{array}{c}\text { IC 95\% } \\
\text { Efficacy }\end{array}$ \\
\hline Day 0 & $100 \%(75 / 75)$ & $8.31 \pm 7.8$ & $1-50$ & & \\
Day 30 & $50.7 \%(38 / 75)$ & $2.28 \pm 3.56$ & $1-14$ & 72.6 & $57.3-82.4$ \\
Day 60 & $34.7 \%(26 / 75)$ & $0.96 \pm 1.74$ & $1-8$ & 88.4 & $81.0-93.0$ \\
Day 90 & $24 \%(18 / 75)$ & $0.51 \pm 1.26$ & $1-8$ & 93.9 & $88.4-96.8$
\end{tabular}


mals had dermatological lesions and flea allergy dermatitis was diagnosed clinically in 12 cats, but these prevalences were reduced to 5 and 3 cats respectively on day 90. Pair comparisons among sampling periods were all statistically significant $(\mathrm{P}<0.05)$, excepting reduction of dermatological lesions between days 60 to 90 (Chi square $=0.86$; $\mathrm{P}=$ 0.354 ) and reduction of FAD lesions between days 0 to 30 (Chi square $=1.14 ; \mathrm{P}=0.286$ ) and between days 60 to 90 (Chi square $=0.35 ; \mathrm{P}=0.555$ ). Similarly, the numbers of cats with bites or scratching were also reduced from 14 to 2 and from 35 to 5 respectively. All pair comparisons being also significant $(\mathrm{P}<0.05)$ excepting for cats with bites between days 0 to 30 (Chi square $=1.85 ; \mathrm{P}=0,174$ ) and scratching prevalence between days 60 to 90 (Chi square $=3.43 ; \mathrm{P}=0.064)$. Finally, the number of cats with flea feces was reduced from 70 , on day 0 , to 14 on day 90 . In this case, all pair comparisons were statistically significant too, excepting between days 30 to 60 (Chi square $=2.2 ; \mathrm{P}=0.138$ ).

\section{DISCUSSION}

Ctenocephalides felis is the predominant species on cats, as reported in other studies carried out in different countries (Cadiergues et al. 2000, Beck et al. 2006, Bond et al. 2007) including Spain (Gracia et al. 2013). The sticktight flea, Echidnophaga gallinacea, is a species frequently found in birds but only occasionally in dogs and cats (Beaucournu \& Launay 1990). Even though this species was not found in a previous study carried out in Spain (Gracia et al. 2013) we found it, in very low number, in a warm area (The Canary Islands).

Flea abundance at Day 0 was associated with hair type, location of the household and time elapsed since the last treatment. A higher infestation in cats with long hair could be explained by the fact that long hair avoids flea capture during host grooming. Fleas were less abundant on cats living in an urban zone compared to those living in a rural/suburban zone which is in agreement with Farkas et al. (2009). Cats in rural areas live under epidemiological conditions favoring heavy flea infestation (Kaal et al. 2006). The abundance of flea species in cats was mainly associated with host habitat and environmental factors rather than host-dependent factors (Gracia et al. 2013) and studies have showed that the main factor affecting this is the life style (indoor/outdoor) and contact with other individuals (Chin et al. 2005, Dryden et al. 2011b), but our analysis, despite specifically studying this circumstance, could not back up it. Another factor that affected the flea rate was the time elapsed from the last insecticidal treatment. Only cats untreated for, at least, two months before the start of the study were included. It is probable that the lower abundance of fleas was due to the consequences on the environmental flea contamination achieved by the previous treatments (Dryden et al. 2011a).

The results of this work show that Frontline Combo ${ }^{\circledR}$ gives an appropriate control of fleas. Treatment controls the natural flea infestations significantly over the duration of the trial. Following three monthly application, cat flea burdens were reduced by $93.9 \%$. The treatment was effective in all cats irrespective of their mode of life.
At the end of the study some cats still hosted some fleas, even though the parasitic burden was remarkably lower in these animals, since only one animal hosted more than 5 fleas on day 90 while 79 raised that burden on day 0 . Our hypothesis is that prior to cat treatment, massive numbers of flea eggs were laid in the indoor and outdoor environments and therefore the flea biomass (especially pupae) was able to continue to allow flea emergence during several weeks or months (Bitam et al. 2010). Efficacy improved over time as treatments reduced the environmental reservoir. In this respect we must point out that not environmental control or treatment of in-contact cats in multicat households was recommended. In addition, due to the ambulant habits of some cats, they might be able to contact sporadically with new infection sources far from home. In the current work, there were no untreated control groups which would have had a high suitability to get to know the natural evolution of flea populations. Unfortunately, as the study involved scattered populations of cats over periods that differed among them in location and availability of animals, this study design using control groups was unavailable.

In order to mimic as much as possible the natural parasitation status of the animal and the real situation of a treated infestation, the fleas were put back on the cat. Although the decision to removal and replace the fleas on the animal could be seen as an intervention that had a negative impact on the animal welfare and/or natural parasitation (damaged of fleas), we want to point out that what is pretended was only quantify the levels of parasitation in the sampling dates, this is not performed any experimental infection; and all surveys were carried out with extreme care to avoid damaging insects.

Only a Frontline Combo $^{\circledR}$ treatment approximately halves the number of cats with dermatological lesions and abrasions related to the presence of fleas (typical FAD sores, scratching and flea bites) as well as the number of cats with flea faeces. Obsessive scratching is the clearest indication that a cat is infested with fleas. In our work, we can see that already in the first sampling this symptom had decreased statistically. In addition, a significant proportion of cats reduced all the symptoms related to the presence of fleas already from the second sampling. These data shows that the treatment with Frontline Combo ${ }^{\circledR}$ was associated with a decrease of the clinical signs related to presence of fleas.

Although direct comparison with other studies is difficult, our results are similar to those obtained by other authors (Schnieder et al. 2008, Beugnet \& Franc 2010, Dryden et al. 2011-a) indicating a good level of efficacy for fipronil 13 years after its commercial launch in veterinary medicine. This study found no evidence of flea resistance to Fipronil/(S)-methoprene

\section{CONCLUSIONS}

A high level of flea control and a remission of the clinical signs related to presence of fleas were observed on cats following 3 monthly application of Frontline Combo ${ }^{\circledR}$.

Pet owners should be warned that it is not unusual to detect some fleas on treated pets for 2-3 months after tre- 
atment. This appears to be particularly true in geographic areas with naturally high levels of flea infestations, like Spain.

Acknowledgements.- The authors would like to thank Merial, Lyon, France for their cooperation and support throughout the Project, also veterinarians for collecting samples.

Disclaimer-- This document is provided for scientific purposes only. Any reference to a brand or trademark herein is for informational purposes only and is not intended for a commercial purpose or to dilute the rights of the respective owner(s) of the brand(s) or trademark(s).

\section{REFERENCES}

Beaucournu J.C. \& Launay H. 1990. Les Puces de France et du Bassin Méditerranéen Occidental. Fédération Française des Sociétés de Sciences Naturelles, París. 548p.

Beck W., Boch K., Mackensen H., Wiegand B. \& Pfister K. 2006. Qualitative and quantitative observation on the flea population dynamics of dogs and cats in several areas of Germany. Vet. Parasitol. 137:130-136.

Beugnet F. \& Franc M. 2010. Results of a European multicentric field efficacy study of fipronil/(S) methoprene combination on flea infestation of dogs and cats during 2009 summer. Parasite 17:337-342.

Bitam I., Dittmar K., Parola P., Whiting M.F. \& Raoult D. 2010. Fleas and flea-borne diseases: a review. Int. J. Infect. Dis. 14:e667-e676.

Bond R., Riddle A., Mottram L., Beugnet F. \& Stevenson R. 2007. Survey of flea infestation in dogs and cats in the United Kingdom during 2005. Vet. Rec. 160:503-506.

Bossard R.L., Dryden M.W. \& Broce A.B. 2002. Insecticide susceptibility of cat fleas (Siphonaptera: Pulicidae) from several regions of the United States. J. Med. Entomol. 39:742-746.

Cabin R.J. \& Mitchell R.J. 2000. To Bonferroni or not to Bonferroni: when and how are the questions. Bull. Ecol. Soc. Am. 81:246-248.

Cadiergues M.C., Deloffre P. \& Franc M. 2000. Répartition des espèces de puces rencontrées chez le chat en France. Revue Med. Vet. 151:447-450.

Chin A., Lunn P. \& Dryden M.W. 2005. Persistent flea infestations in dogs and cats controlled with monthly topical applications of fipronil and methoprene. Aust. Vet. Pract. 35:89-96.

Chomel B.B., Boulouis H.J., Maruyama S. \& Breitschwerdt E.B. 2006. Bartonella spp. in pets and effect on human health. Emerg. Infect. Dis. 12:389-394.

Coles G.C., Bauer C., Borgsteede F.H.M., Geerts S., Klei T.R., Taylor M.A. \& Waller P.J. 1992. World Association for the Advancement of Veterinary Parasitology (WAAVP) methods for the detection of anthelmintic resistance in nematodes of veterinary importance. Vet. Parasitol. 44:35-44.

Dryden M., Payne P. \& Smith V. 2007. Efficacy of selamectin and fipronil/
(S)-methoprene spot-on formulations applied to cats against adult cat fleas (Ctenocephalides felis), flea eggs, and adult flea emergence. Vet. Ther. 8:255-262.

Dryden M., Payne P., Smith V., Riggs B., Davenport J. \& Kobuszewski D. 2011-a. Efficacy of dinotefuran-pyriproxyfen-permethrin and fipronil/ (S)-methoprene topical spot-on formulations to control flea populations in naturally infested pets and private residences in Tampa, FL. Vet. Parasitol. 182:281-286.

Dryden M., Carithers D., McBride A., Riggs B., Smith L., Davenport J., Smith V., Payne P. \& Gross S. 2011-b. A comparison of flea control measurement methods for tracking flea populations in highly infested private residences in Tampa FL, following topical treatment of pets with Frontline ${ }^{\circledR}$ Plus (Fipronil/(S)-Methoprene). Int. J. Appl. Res. Vet. Med. 9:356-367.

Farkas R., Gyurkovszky M., Solymosi N. \& Beugnet F. 2009. Prevalence of flea infestation in dogs and cats in Hungary combined with a survey of owner awareness. Med. Vet. Entomol. 23:187-194.

Gracia M.J., Calvete C., Estrada R., Castillo J.A., Peribáñez M.A. \& Lucientes J. 2013. Survey of flea infestation in cats in Spain. Med. Vet. Entomol. 27:175-180.

Kaal J.F., Baker K. \& Torgerson P.R. 2006. Epidemiology of flea infestation of ruminants in Libya. Vet. Parasitol. 141:313-318.

Marchiondo A.A., Holdsworth P.A., Green P., Blagburn B.L. \& Jacobs D.E. 2007. World Association for the Advancement of Veterinary Parasitology (WAAVP) guidelines for evaluating the efficacy of parasiticides for the treatment, prevention and control of flea and tick infestation on dogs and cats. Vet. Parasitol. 3/4:332-344.

Mardia K.V., Kent J.T. \& Bibby J.M. 1979. Multivariate Analysis. Academic Press, London. ISBN 0-12-471252-5. 521p.

Narahashi T., Zhao X., Ikeda T., Salgado V.L. \& Yeh J.Z. 2010. Glutamateactivated chloride channels: Unique fipronil targets present in insects but not in mammals. Pest Biochem. Physiol. 97:149-152.

Narahashi T., Zhao X., Ikeda T., Nagata K. \& Yeh J.Z. 2007. Differential actions of insecticides on target sites: basis for selective toxicity. Hum. Exp. Toxicol. 26:361-366.

Pérez-Osorio C.E., Zavala-Velázquez J.E., Arias-León J.J. \& Zavala-Castro J.E. 2008. Rickettsia felis as emergent global threat for humans. Emerg. Infect. Dis. 14:1019-1023.

Schnieder T., Wolken S. \& Mencke N. 2008. Comparative efficacy of imidacloprid, selamectin, fipronil/(S)-methoprene, and metaflumizone against cats experimentally infested with Ctenocephalides felis. Vet. Ther. 9:176-183.

Tasker S. 2010. Haemotropic mycoplasmas: what's their real significance in cats? J. Feline Med. Surg. 12:369-381.

Young D.R., Jeannin P.C. \& Boeckh A. 2004. Efficacy of fipronil/(S)-methoprene combination spot-on for dogs against shed eggs, emerging and existing adult cat fleas (Ctenocephalides felis, Bouché). Vet. Parasitol. 125:397-407. 